PROCEEDINGS OF THE AMERICAN MATHEMATICAL SOCIETY

Volume 126, Number 4, April 1998, Pages 1023-1029

S 0002-9939(98)04009-X

\title{
EXTENDIBILITY OF HOMOGENEOUS POLYNOMIALS ON BANACH SPACES
}

\author{
PÁDRAIG KIRWAN AND RAYMOND A. RYAN
}

(Communicated by Theodore W. Gamelin)

\begin{abstract}
We study the $n$-homogeneous polynomials on a Banach space $X$ that can be extended to any space containing $X$. We show that there is an upper bound on the norm of the extension. We construct a predual for the space of all extendible $n$-homogeneous polynomials on $X$ and we characterize the extendible 2-homogeneous polynomials on $X$ when $X$ is a Hilbert space, an $\mathcal{L}_{1}$-space or an $\mathcal{L}_{\infty}$-space.
\end{abstract}

\section{INTRODUCTION}

There is no Hahn-Banach theorem for $n$-homogeneous polynomials on a Banach space when $n>1$. For example, the 2-homogeneous polynomial $P(x)=\sum_{k} x_{k}^{2}$ on $l_{2}$ cannot be extended to any $C(K)$ space containing $l_{2}$, as the presence of the Dunford-Pettis property on $C(K)$ would force $P$ to be weakly sequentially continuous [1]. Even when a homogeneous polynomial can be extended to a larger space, it can happen that the norm cannot be preserved [2, 9]. The Aron-Berner extension process for homogeneous polynomials on a Banach space $X$ [2] avoids these obstacles by requiring that the extension be defined only for a very restricted class of spaces containing $X$ - essentially, the bidual $X^{* *}$ and spaces closely related to it (see also $[1,4,7,10,13]$ ). A similar observation applies to the ultrapower method of Dineen-Timoney and Lindström-Ryan [6, 8].

We propose to study those homogeneous polynomials on a Banach space $X$ that extend to every space containing $X$. We do not require that the norm be preserved. We show that, if a homogeneous polynomial, $P$, on $X$ can be extended to every space containing $X$, then it is possible to put an upper bound on the norms of the extensions. This enables us to put a natural norm on the space $\mathcal{P}_{e}\left({ }^{n} X\right)$ of "extendible" $n$-homogeneous polynomials. We show that $\mathcal{P}_{e}\left({ }^{n} X\right)$ is complete in this norm. We then construct a predual for the Banach space $\mathcal{P}_{e}\left({ }^{n} X\right)$. We conclude with some special classes of spaces. We characterize the extendible 2-homogeneous polynomials on $X$ when $X$ is a Hilbert space, an $\mathcal{L}_{1}$-space or an $\mathcal{L}_{\infty}$-space.

All the Banach spaces considered can be taken over the real or complex numbers. $\mathcal{P}\left({ }^{n} X ; Y\right)$ denotes the Banach space of bounded, $n$-homogeneous polynomials from $X$ into $Y$. Thus if $P \in \mathcal{P}\left({ }^{n} X ; Y\right)$, then there exists a unique bounded symmetric multilinear mapping $A: X^{n} \rightarrow Y$ such that $P(x)=A(x, \ldots, x)$ for every $x \in X$.

Received by the editors May 17, 1996 and, in revised form, July 10, 1996.

1991 Mathematics Subject Classification. Primary 46G20; Secondary 46B28.

Key words and phrases. Homogeneous polynomial, extendibility.

(C)1998 American Mathematical Society 
When $Y$ is the scalar field we denote this space by $\mathcal{P}\left({ }^{n} X\right)$. For $P \in \mathcal{P}\left({ }^{n} X\right)$ we can define a bounded linear operator $T: X \rightarrow \mathcal{P}\left({ }^{n-1} X\right)$ by $T(x)(y)=A(x, y, \ldots, y)$, where $A$ is the multilinear form on $X^{n}$ that generates $P$ as described above. We shall refer to $T$ as the linear operator associated with $P$.

\section{EXTENDIBILITY OF LINEAR OPERATORS AND HOMOGENEOUS POLYNOMIALS}

We begin with the description of a process that will enable us to "paste together" a family of spaces containing $X$ in a coherent way. Let $j_{\alpha}: X \rightarrow Z_{\alpha}$ be a family of embeddings, indexed by $\alpha \in A$. By an amalgamation of this family we shall mean a triple consisting of a Banach space $Z$, an embedding $j: X \rightarrow Z$ and a family of bounded linear operators $i_{\alpha}: Z_{\alpha} \rightarrow Z$ with $\left\|i_{\alpha}\right\| \leq 1$, such that $i_{\alpha} \circ j_{\alpha}=j$ for every $\alpha \in A$. Where there is no danger of confusion, we shall refer to the amalgamation $\left(Z, j,\left\{i_{\alpha}\right\}\right)$ simply as "the amalgamation $Z$ ".

An amalgamation may be constructed as follows: first, form the $l_{1}$-sum $\left(\sum_{\alpha} Z_{\alpha}\right)_{1}$ and let $k_{\alpha}$ denote the usual embedding of $Z_{\alpha}$ into this space. Let

$$
N=\left\{\left(j_{\alpha} x_{\alpha}\right) \in\left(\sum_{\alpha} Z_{\alpha}\right)_{1}: x_{\alpha} \in X, \sum_{\alpha} x_{\alpha}=0\right\} .
$$

It is easy to see that $N$ is a closed subspace of $\left(\sum_{\alpha} Z_{\alpha}\right)_{1}$. Let $Z$ be the quotient space:

$$
Z=\left(\sum_{\alpha} Z_{\alpha}\right)_{1} / N
$$

and let $\pi$ be the quotient mapping of $\left(\sum_{\alpha} Z_{\alpha}\right)_{1}$ onto $Z$. For each $\alpha \in A$ let $i_{\alpha}=\pi \circ k_{\alpha}$. Thus, we have $\left\|i_{\alpha}\right\| \leq 1$ for every $\alpha$. To define an embedding of $X$ into $Z$ we note first that for all $\alpha, \beta \in A$, we have $k_{\alpha} j_{\alpha}(x)-k_{\beta} j_{\beta}(x) \in N$ for every $x \in X$. Therefore $\pi k_{\alpha} j_{\alpha}(x)=\pi k_{\beta} j_{\beta}(x)$ and we can define

$$
j(x)=\pi k_{\alpha} j_{\alpha}(x) \quad \text { for any } \alpha \in A .
$$

To see that $j$ is an embedding, let $x \in X$. It is clear from the definition of $j(x)$ that $\|j(x)\| \leq\|x\|$. On the other hand, if we choose any $\alpha \in A$, we have, by the definition of the quotient norm,

$$
\|j(x)\|=\inf \left\{\left\|k_{\alpha} j_{\alpha}(x)+u\right\|: u \in N\right\} .
$$

Now if $u \in N$, then $u=\left(j_{\beta} x_{\beta}\right)$ where $\sum_{\beta} x_{\beta}=0$ and so

$$
\begin{aligned}
\left\|k_{\alpha} j_{\alpha}(x)+u\right\| & =\left\|j_{\alpha}(x)+j_{\alpha}\left(x_{\alpha}\right)\right\|+\sum_{\beta \neq \alpha}\left\|j_{\beta}\left(x_{\beta}\right)\right\| \\
& =\left\|x+x_{\alpha}\right\|+\sum_{\beta \neq \alpha}\left\|x_{\beta}\right\| \geq\left\|x+x_{\alpha}+\sum_{\beta \neq \alpha} x_{\beta}\right\| \\
& =\left\|x+\sum_{\beta} x_{\beta}\right\|=\|x\|,
\end{aligned}
$$

since $\sum x_{\beta}=0$. Therefore $\|j(x)\|=\|x\|$ for every $x \in X$. Finally, it follows from the definition of $j$ that $i_{\alpha} \circ j_{\alpha}=j$ for every $\alpha$. Thus $\left(Z, j,\left(j_{\alpha}\right)\right)$ meets all the requirements for an amalgamation of the family of embeddings $\left(j_{\alpha}\right)$. 
We shall say that a bounded $n$-homogeneous polynomial $P: X \rightarrow Y$ is extendible if, for every embedding of $X$ into a Banach space $Z$, there exists an extension of $P$ to a bounded $n$-homogeneous polynomial $Q: Z \rightarrow Y$. With the help of amalgamations, we can show that we can control the norm of the extension independently of the space $Z$ :

Proposition 1. If $P \in \mathcal{P}\left({ }^{n} X ; Y\right)$ is extendible, then there exists $C>0$ such that for every embedding of $X$ into a Banach space $Z$, there is an extension $Q \in$ $\mathcal{P}\left({ }^{n} Z ; Y\right)$ of $P$ with $\|Q\| \leq C$.

Proof. If this were false, then for every $n \in \mathbf{N}$ there would exist a Banach space $Z_{n}$ and an embedding $j_{n}: X \rightarrow Z_{n}$ such that every extension of $P$ to $Z_{n}$ has norm at least $n$. Let $\left(Z, j,\left(i_{n}\right)\right)$ be an amalgamation of this sequence of embeddings. Now $P$ has a bounded extension, $Q$, defined on $Z$. But then $Q \circ i_{n}$ is an extension of $P$ to $Z_{n}$, and hence

$$
\|Q\| \geq\left\|Q \circ i_{n}\right\| \geq n \quad \text { for every } n \in \mathbf{N},
$$

which is impossible. This concludes the proof.

Let $\mathcal{P}_{e}\left({ }^{n} X ; Y\right)$ denote the subset of $\mathcal{P}\left({ }^{n} X ; Y\right)$ consisting of all the extendible $n$-homogeneous polynomials. $\mathcal{P}_{e}\left({ }^{n} X ; Y\right)$ is a vector space on which we can define a norm by letting $e(P)$ be the smallest positive real number $C$ with the property that for every space $Z$ containing $X$ there is an extension $Q \in \mathcal{P}\left({ }^{n} Z ; Y\right)$ of $P$ with $\|Q\| \leq C$. We have $\|P\| \leq e(P)$ for every $P \in \mathcal{P}_{e}\left({ }^{n} X ; Y\right)$.

Proposition 2. $\left(\mathcal{P}_{e}\left({ }^{n} X ; Y\right), e\right)$ is complete.

Proof. It suffices to show that every absolutely summable series is convergent. Accordingly, suppose that $\sum_{k} e\left(P_{k}\right)<\infty$, where $P_{k} \in \mathcal{P}_{e}\left({ }^{n} X ; Y\right)$ for every $k$. Then $\sum P_{k}$ is absolutely summable in $\mathcal{P}^{n}(X ; Y)$ and hence it converges in this space to a bounded $n$-homogeneous polynomial $P$. Let $Z$ be a space that contains $X$. For each $k$ there exists an extension $Q_{k}$ of $P_{k}$ to $Z$, with $\left\|Q_{k}\right\| \leq e\left(P_{k}\right)$. It follows that the series $\sum Q_{k}$ converges in $\mathcal{P}\left({ }^{n} Z ; Y\right)$ to a polynomial $Q$. It is clear that $Q$ extends $P$, and so $P \in \mathcal{P}_{e}\left({ }^{n} X ; Y\right)$. Furthermore, we have $\|Q\| \leq \sum\left\|Q_{k}\right\| \leq \sum e\left(P_{k}\right)$. Finally, we have

$$
e\left(P-\sum_{k=1}^{N} P_{k}\right)=e\left(\sum_{k>N} P_{k}\right) \leq \sum_{k>N} e\left(P_{k}\right),
$$

and hence $\sum P_{k}$ converges to $P$ in $\left(\mathcal{P}_{e}\left({ }^{n} X ; Y\right), e\right)$. This concludes the proof.

We shall see that the uniform norm is equivalent to the norm $e$ on $\mathcal{P}_{e}\left({ }^{n} X\right)$ if and only if every polynomial in $\mathcal{P}\left({ }^{n} X\right)$ is extendible.

\section{The PREDUAL of the SPACE OF EXTEndible POlynomials}

We begin with the construction of a predual for the space $\mathcal{L}_{e}\left({ }^{2} X \times Y\right)$ of extendible bilinear forms on $X \times Y$. We can define a complete norm, $e$, on this space exactly as in the previous section. We shall denote the projective norm on the tensor product $X \otimes Y$ by $\pi_{X, Y}$. We refer the reader to [3] for further details on tensor products. Now if $i, j$ are embeddings of $X, Y$ into $W, Z$ respectively, then $i \otimes j$ is an algebraic embedding of $X \otimes Y$ into $W \otimes Z$. Thus we may identify $X \otimes Y$ with a vector subspace of $W \otimes Z$. For $u \in X \otimes Y$ we have $\pi_{W, Z}(u) \leq \pi_{X, Y}(u)$. The norms $\pi_{W, Z}$ and $\pi_{X, Y}$ are equivalent on $X \otimes Y$ if and only if every bounded 
bilinear form on $X \times Y$ has a bounded extension to $W \times Z$. For an element $u$ of $X \otimes Y$, we define

$$
\eta(u)=\inf \left\{\pi_{W, Z}(u): X \subset W, Y \subset Z\right\},
$$

the infimum being taken over all pairs of embeddings $X \hookrightarrow W, Y \hookrightarrow Z$. We claim that $\eta$ is a reasonable crossnorm on $X \otimes Y$. Obviously, we have $\eta(\lambda u)=|\lambda| \eta(u)$. To see that $\eta$ is subadditive, let $u_{1}, u_{2} \in X \otimes Y$ and let $\varepsilon>0$. For each $r=1,2$ there exists a pair of embeddings $i_{r}: X \hookrightarrow W_{r}, j_{r}: Y \hookrightarrow Z_{r}$, such that $\pi_{W_{r}, Z_{r}}\left(u_{r}\right) \leq$ $\eta\left(u_{r}\right)+\varepsilon / 2$. Let $\left(W, i,\left(k_{r}\right)\right),\left(Z, j,\left(l_{r}\right)\right)$ respectively be amalgamations of these embeddings. Then we have $i \otimes j=\left(k_{r} \otimes l_{r}\right) \circ\left(i_{r} \otimes j_{r}\right)$ for each $r$ and since $\left\|k_{r}\right\|$, $\left\|l_{r}\right\| \leq 1$ it follows that $\pi_{W, Z} \leq \pi_{W_{r}, Z_{r}}$ for each $r$. Therefore

$$
\eta\left(u_{1}+u_{2}\right) \leq \pi_{W, Z}\left(u_{1}+u_{2}\right) \leq \pi_{W, Z}\left(u_{1}\right)+\pi_{W, Z}\left(u_{2}\right) \leq \eta\left(u_{1}\right)+\eta\left(u_{2}\right)+\varepsilon,
$$

and hence $\eta\left(u_{1}+u_{2}\right) \leq \eta\left(u_{1}\right)+\eta\left(u_{2}\right)$. Next, suppose that $\eta(u)=0$ for some $u \in X \otimes Y$. Then there is a sequence of pairs of embeddings $X \hookrightarrow W_{r}, Y \hookrightarrow Z_{r}$ such that $\pi_{W_{r}, Z_{r}}(u)<1 / r$. Arguing as above, let $W, Z$ be amalgamations of these sequences. Then we have $\pi_{W, Z}(u) \leq \pi_{W_{r}, Z_{r}}(u)<1 / r$ for every $k$ and so $\pi_{W, Z}(u)=0$, whence $u=0$. This shows that $\eta$ is a norm. To see that $\eta$ is a reasonable crossnorm, note first that if $x \in X, y \in Y$, then $\pi_{W, Z}(x \otimes y)=\|x\|\|y\|$ for all $W, Z$ and so $\eta(x \otimes y)=\|x\|\|y\|$. Secondly, let $\varphi \in X^{*}, \psi \in Y^{*}$. Let $u \in X \otimes Y$ with $\eta(u)<1$. Choose embeddings $X \hookrightarrow W, Y \hookrightarrow Z$ such that $\pi_{W, Z}(u)<1$ and let $\varphi_{W}, \psi_{Z}$ be Hahn-Banach extensions of $\varphi, \psi$ to $W, Z$ respectively. Then

$$
|\varphi \otimes \psi(u)|=\left|\varphi_{W} \otimes \psi_{Z}(u)\right| \leq\left\|\varphi_{W}\right\|\left\|\psi_{Z}\right\|=\|\varphi\|\|\psi\| .
$$

It follows that $\eta$ is a reasonable crossnorm.

Proposition 3. $\left(X \hat{\otimes}_{\eta} Y\right)^{*}$ is isometrically isomorphic to $\mathcal{L}_{e}\left({ }^{2} X \times Y\right)$.

Proof. Every bounded linear functional on $X \hat{\otimes}_{\eta} Y$ is the linearization, $\widetilde{T}$, of a bounded bilinear form $T$ on $X \times Y$. Since $\widetilde{T}$ is $\eta$-continuous, we have $|\widetilde{T}(u)| \leq$ $\eta^{*}(\widetilde{T}) \eta(u) \leq \eta^{*}(\widetilde{T}) \pi_{W, Z}(u)$ for every $u \in X \otimes Y$ and every pair of embeddings $X \hookrightarrow W, Y \hookrightarrow Z$. Hence $\widetilde{T}$ is continuous on $X \otimes Y$ for the norm induced by $W \otimes_{\pi} Z$ and it follows from the Hahn-Banach theorem that $T$ extends to a bilinear form $S$ on $W \times Z$ with $\|S\| \leq \eta^{*}(\widetilde{T})$. Therefore $T$ is extendible and $e(T) \leq \eta^{*}(\widetilde{T})$. Conversely, if $T \in \mathcal{L}_{e}\left({ }^{2} X \times Y\right)$ and $X \hookrightarrow W, Y \hookrightarrow Z$, then $T$ extends to $S \in \mathcal{L}\left({ }^{2} W \times Z\right)$ with $\|S\| \leq e(T)$. It follows that $|\widetilde{T}(u)|=|\widetilde{S}(u)| \leq e(T) \pi_{W, Z}(u)$ for $u \in X \otimes Y$ and hence $\widetilde{T}$ is $\eta$-continuous, with $\eta^{*}(\widetilde{T}) \leq e(T)$. Therefore $\eta^{*}(\widetilde{T})=e(T)$. This completes the proof.

We now construct a predual for the space of extendible $n$-homogeneous polynomials. The dual of the $n$-fold symmetric tensor product $\widehat{\bigotimes}_{s, \pi}^{n} X$ is the Banach space $\mathcal{L}_{s}\left({ }^{n} X\right)$ of symmetric, continuous $n$-linear forms on $X$. Since this latter space is isomorphic to $\mathcal{P}\left({ }^{n} X\right)$, it follows that $\widehat{\bigotimes}_{s, \pi}^{n} X$ can be considered as an "isomorphic" predual of $\mathcal{P}\left({ }^{n} X\right)$. We prefer to renorm $\widehat{\bigotimes}_{s, \pi}^{n} X$ so this becomes an isometry, as follows: for $u \in \widehat{\bigotimes}_{s, \pi} X$, we will define

$$
\|u\|_{\pi}=\sup \left\{|A(u)|: A \in \mathcal{L}_{s}\left({ }^{n} X\right) \text { and }\|\widehat{A}\|=1\right\},
$$

where $\widehat{A}$ denotes the $n$-homogeneous polynomial associated with $A$. This norm is equivalent to the projective norm on $\widehat{\bigotimes}_{s, \pi}^{n} X$. Let us write $x^{n}$ for the tensor 
$x \otimes x \otimes \cdots \otimes x$. Then, if $u$ belongs to the uncompleted symmetric tensor product $\bigotimes_{s, \pi}^{n} X$, it follows from the polarization formula that $u$ can be expressed as a linear combination of tensors of the form $x^{n}$. We then have another formula for $\|u\|_{\pi}$ :

$$
\|u\|_{\pi}=\inf \left\{\sum_{j=1}^{m}\left|\lambda_{j}\right|\left\|x_{j}\right\|^{n}: u=\sum_{j=1}^{m} \lambda_{j} x_{j}^{n}\right\} .
$$

We shall denote by $\widehat{X}_{\pi}^{(n)}$ the space $\widehat{\bigotimes}_{s, \pi}^{n} X$ with this equivalent norm. Then $\mathcal{P}\left({ }^{n} X\right)$ is the dual space of $\widehat{X}_{\pi}^{(n)}$. The mapping $x \mapsto x^{n}$ is a "universal" continuous $n$-homogeneous polynomial on $X$ : for every $P \in \mathcal{P}\left({ }^{n} X\right)$ there is a unique $\widetilde{P} \in$ $\left(\widehat{X}_{\pi}^{(n)}\right)^{*}$ with the same norm, such that

$$
P(x)=\widetilde{P}\left(x^{n}\right) \text { for every } x \in X .
$$

We refer to $[11,12]$ for further details.

Now the space $\widehat{X}_{\pi}^{(n)}$ suffers the same defect as the projective tensor product $X \otimes Y$ in that its norm does not respect embeddings of $X$ into larger spaces. Proceeding as we did for $X \otimes Y$, we can define a norm, $\eta$, on $X^{(n)}$ by

$$
\eta(u)=\inf \left\{\sum_{j=1}^{m}\left|\lambda_{j}\right|\left\|w_{j}\right\|^{n}: u=\sum_{j=1}^{m} \lambda_{j} w_{j}^{n}, w_{j} \in W, X \subset W\right\},
$$

the infimum being taken over all embeddings $X \hookrightarrow W$. We denote by $X_{\eta}^{(n)}$ the homogeneous product space $X^{(n)}$ with this norm, and we denote the completion of this space by $\widehat{X}_{\eta}^{(n)}$. We then have $\left(\widehat{X}_{\eta}^{(n)}\right)^{*}=\mathcal{P}_{e}\left({ }^{n} X\right)$ :

Proposition 4. $P \in \mathcal{P}\left({ }^{n} X\right)$ is extendible if and only if the linear form $\widetilde{P}$ on $X^{(n)}$ is continuous with respect to the norm $\eta$. The correspondence $P \leftrightarrow \widetilde{P}$ is an isometric isomorphism of $\left(\mathcal{P}_{e}\left({ }^{n} X\right), e\right)$ with $\left(\widehat{X}_{\eta}^{(n)}\right)^{*}$.

The proof is similar to the proof of Proposition 3. D. Cardano and I. Zalduendo have shown that integral polynomials are extendible. This also follows from the above proposition - the $n$-homogeneous polynomial $P$ is integral precisely when the linear form $\widetilde{P}$ is continuous with respect to the injective norm $\varepsilon$ and since $\eta$ is a reasonable crossnorm, we have $\varepsilon \leq \eta$.

Corollary 5. The norm $e$ is equivalent to the uniform norm on $\mathcal{P}_{e}\left({ }^{n} X\right)$ if and only if every $n$-homogeneous polynomial on $X$ is extendible.

Proof. If $\mathcal{P}\left({ }^{n} X\right)=\mathcal{P}_{e}\left({ }^{n} X\right)$, then, since $\|\cdot\| \leq e$, it follows that these norms are equivalent. Conversely, suppose these norms are equivalent on $\mathcal{P}_{e}\left({ }^{n} X\right)$. Since the uniform norm is the dual of the projective norm and $e$ is the dual of $\eta$, it follows that the projective norm is equivalent to $\eta$ on $X^{(n)}$. Therefore these norms yield the same dual, hence $\mathcal{P}\left({ }^{n} X\right)=\mathcal{P}_{e}\left({ }^{n} X\right)$. This concludes the proof.

Thus, if every $n$-homogeneous polynomial on $X$ is extendible, then there exists a positive constant $C$, depending only on $X$ and $n$, such that for every embedding $X \hookrightarrow Z$, every $P \in \mathcal{P}\left({ }^{n} X\right)$ has an extension to $Z$ whose norm is at most $C\|P\|$. On the other hand, if $X$ supports an $n$-homogeneous polynomial that is not extendible, then, using an amalgamation argument, we see that there is an embedding $X \hookrightarrow Z$ and a sequence of $n$-homogeneous polynomials $P_{k}$ on $X$ such that every extension of $P_{k}$ to $Z$ has norm at least equal to $k$. 


\section{EXAMPLES}

We begin with a simple observation:

Lemma 6. If $P \in \mathcal{P}\left({ }^{2} X\right)$ is extendible, then the associated linear operator $T$ : $X \rightarrow X^{*}$ is 2-summing.

Proof. There exists an indexing set $I$ and an embedding $j: X \rightarrow l_{\infty}^{I}$. Let $Q \in$ $\mathcal{P}\left({ }^{2} l_{\infty}^{I}\right)$ be an extension of $P$ and let $S$ be the associated linear operator. Since every bounded linear operator from an $\mathcal{L}_{\infty}$-space into an $\mathcal{L}_{1}$-space is 2 -summing, $S$ is 2 -summing. Hence $T=j^{*} \circ S \circ j$ is 2 -summing. This concludes the proof.

In general, this condition is not sufficient for $P$ to be extendible. However, for $\mathcal{L}_{1}$-spaces, it is enough:

Proposition 7. Suppose $X$ is an $\mathcal{L}_{1}$-space. Then $P \in \mathcal{P}\left({ }^{2} X\right)$ is extendible if and only if the associated linear operator $T: X \rightarrow X^{*}$ is 2-summing.

Proof. If $T$ is 2-summing, then there exists a probability measure $\mu$ and a factorization of $T$ as $u \circ J_{2} \circ v$, where $v \in \mathcal{L}\left(X, L_{\infty}(\mu)\right), u \in \mathcal{L}\left(L_{2}(\mu), X^{*}\right)$ and $J_{2}: L_{\infty}(\mu) \rightarrow L_{2}(\mu)$ is the canonical inclusion. Now let $j: X \rightarrow Z$ be an embedding. Since $L_{\infty}(\mu)$ is injective, $v$ extends to an operator $\tilde{v}: Z \rightarrow L_{\infty}(\mu)$ and then $S=u \circ J_{2} \circ \tilde{v}$ extends $T$. Consider the restriction of $S^{*}$ to $X$. We have $\left.S^{*}\right|_{X}=\left.\tilde{v}^{*} \circ J_{2}^{*} \circ u^{*}\right|_{X}$. Now, since $X$ is an $\mathcal{L}_{1}$-space, the operator $\left.u^{*}\right|_{X}: X \rightarrow L_{2}(\mu)$ is 1-summing, and hence 2 -summing. Therefore this operator extends to an operator $w: Z \rightarrow L_{2}(\mu)$. Let $R: Z \rightarrow Z^{*}$ be the composition $\tilde{v}^{*} \circ J_{2}^{*} \circ w$ and let $Q$ be the 2-homogeneous polynomial on $l_{\infty}^{I}$ associated with $R$. Then $Q$ extends $P$. This concludes the proof.

Next, we consider linear operators and 2-homogeneous polynomials on a Hilbert space $H$. The situation concerning extendibility of linear operators on Hilbert spaces is particularly simple. In general, 2-summing operators are extendible, since they factor through $L_{\infty}(\mu)$ spaces [5]. Conversely, suppose that $T: H \rightarrow H$ is extendible. Let $j: H \rightarrow l_{\infty}^{I}$ be an embedding. $T$ extends to an operator $S: l_{\infty}^{I} \rightarrow H$. But every such operator is 2 -summing, and hence $T$ is 2 -summing. Therefore, the spaces of extendible and 2 -summing operators on $H$ coincide.

The situation with 2-homogeneous polynomials is different. Let $P$ be an extendible 2-homogeneous polynomial on $H$ and let $T: H \rightarrow H$ be the associated linear operator. Let $j: H \rightarrow l_{\infty}^{I}$ be an embedding. $P$ extends to a 2-homogeneous polynomial $Q$ on $l_{\infty}^{I}$ which has an associated linear operator $S: l_{\infty}^{I} \rightarrow\left(l_{\infty}^{I}\right)^{*}$ with the property that $j^{*} \circ S \circ j=T$. Since $\left(l_{\infty}^{I}\right)^{*}$ is an $\mathcal{L}_{1}$-space, $S: l_{\infty}^{I} \rightarrow\left(l_{\infty}^{I}\right)^{*}$ and $j^{*}:\left(l_{\infty}^{I}\right)^{*} \rightarrow H$ are both 2 -summing, and hence $j^{*} \circ S$ is nuclear [5, p. 119]. Therefore $T$ is nuclear and it follows that $P$ is also nuclear. Thus every extendible 2-homogeneous polynomial on $H$ is nuclear. On the other hand, it follows easily from the Hahn-Banach theorem that nuclear polynomials are extendible. In summary, we have:

Proposition 8. Let $H$ be a Hilbert space.

(a) A bounded linear operator from $H$ into $H$ is extendible if and only if it is 2-summing.

(b) A bounded 2-homogeneous polynomial on $H$ is extendible if and only if it is nuclear. 
Finally, let $X$ be an $\mathcal{L}_{\infty}$-space. Then $X^{* *}$ is injective and so, by the Aron-Berner extension theorem [2] we have:

Proposition 9 (Aron and Berner). Suppose $X$ is an $\mathcal{L}_{\infty}$-space. Then every $P \in$ $\mathcal{P}\left({ }^{n} X\right)$ is extendible.

\section{REFERENCES}

1. R. Aron, Extension and lifting theorems for analytic mappings, Functional Analysis: Surveys and Recent Results II, Math. Stud. 38, North-Holland, 1980, 257-267. MR 81i:46006

2. R. Aron and P. Berner, A Hahn-Banach extension theorem for analytic mappings, Bull. Soc. Math. France 106 (1978), 3-24. MR 80e:46029

3. A. Defant and K. Floret, Tensor Norms and Operator Ideals, North-Holland Math. Studies 176, 1993. MR 94e:46130

4. A. M. Davie and T. W. Gamelin, A theorem on polynomial-star approximation, Proc. Amer. Math. Soc. 106 (1989), 351-356. MR 89k:46023

5. J. Diestel, H. Jarchow and A. Tonge, Absolutely Summing Operators, Cambridge University Press, 1995. MR 96i:46001

6. S. Dineen and R. Timoney, Complex geodesics on convex domains, Progress in Functional Analysis (ed. K. Bierstedt, J. Bonet, J. Horvath and M. Maestre), Math. Studies 170, NorthHolland, 1992, 333-365. MR 92m:46066

7. P. Galindo, D. García, M. Maestre and J. Mujica, Extension of multilinear mappings on Banach spaces, Studia Math. 108 (1994), 55-76. MR 95f: 46072

8. M. Lindström and R. A. Ryan, Applications of ultraproducts to infinite dimensional holomorphy, Math. Scand. 71 (1992), 229-242. MR 94c:46090

9. P. Mazet, A Hahn-Banach theorem for quadratic forms, preprint.

10. L. Moraes, A Hahn-Banach extension theorem for some holomorphic functions, Complex Analysis, Functional Analysis and Approximation Theory (ed. J. Mujica), Math. Studies 125, North-Holland, 1986, 205-220. MR 88f:46094

11. R. A. Ryan, Applications of Topological Tensor Products to Infinite Dimensional Holomorphy, Ph.D. Thesis, Trinity College, Dublin, 1980.

12. R. A. Ryan and J. B. Turret, Products of linear functionals, Preprint, 1995.

13. I. Zalduendo, A canonical extensions for analytic functions on Banach spaces, Trans. Amer. Math. Soc. 320 (1990), 747-763. MR 90k:46108

Department of Mathematics, University College, Galway, Ireland

Current address: Department of Physical and Quantitative Sciences, Waterford Institute of Technology, Waterford, Ireland

E-mail address: pkirwan@staffmail.wit.ie

E-mail address: ray.ryan@ucg.ie 